\title{
Gerontology
}

\section{Kinect-Based Five-Times-Sit-to-Stand Test for Clinical and In-Home Assessment of Fall Risk in Older People}

\author{
Andreas Ejupi $^{\mathrm{a}-\mathrm{c}}$ Matthew Brodie ${ }^{c}$ Yves J. Gschwind ${ }^{\mathrm{c}}$ Stephen R. Lord ${ }^{c}$ \\ Wolfgang L. Zagler ${ }^{b}$ Kim Delbaere ${ }^{c}$ \\ ${ }^{a}$ Austrian Institute of Technology, Assistive Healthcare Information Technology Group, and bVienna University of \\ Technology, Vienna, Austria; ' Neuroscience Research Australia, University of New South Wales, \\ Sydney, N.S.W., Australia
}

\section{Key Words}

Older adults · Aged $\cdot$ Accidental falls · Assessment .

Kinect-based · Microsoft Kinect · Sit-to-stand · In-home .

Unsupervised $\cdot$ Prediction $\cdot$ Fall risk

\begin{abstract}
Background: Accidental falls remain an important problem in older people. The five-times-sit-to-stand (5STS) test is commonly used as a functional test to assess fall risk. Recent advances in sensor technologies hold great promise for more objective and accurate assessments. Objective: The aims of this study were: (1) to examine the feasibility of a low-cost and portable Kinect-based 5STS test to discriminate between fallers and nonfallers and (2) to investigate whether this test can be used for supervised clinical, supervised and unsupervised in-home fall risk assessments. Methods: A total of 94 community-dwelling older adults were assessed by the Kinect-based 5STS test in the laboratory and 20 participants were tested in their own homes. An algorithm was developed to automatically calculate timing- and speed-related measurements from the Kinect-based sensor data to discriminate between fallers and nonfallers. The associations of these measurements with standard clinical fall risk tests and the results of supervised and unsupervised in-home assessments were examined. Results: Fallers were significantly slower
\end{abstract}

\section{KARGER}

(c) 2015 S. Karger AG, Base

0304-324X/15/0621-0118\$39.50/0

E-Mail karger@karger.com

www.karger.com/ger than nonfallers on Kinect-based measures. The mean velocity of the sit-to-stand transitions discriminated well between the fallers and nonfallers based on 12-month retrospective fall data. The Kinect-based measures collected in the laboratory correlated strongly with those collected in the supervised $(r=0.704-0.832)$ and unsupervised $(r=0.775-0.931)$ in-home assessments. Conclusion: In summary, we found that the Kinect-based 5STS test discriminated well between the fallers and nonfallers and was feasible to administer in clinical and supervised in-home settings. This test may be useful in clinical settings for identifying high-risk fallers for further intervention or for regular in-home assessments in the future.

๑) 2015 S. Karger AG, Basel

\section{Introduction}

Falls remain an important problem for older people with a yearly fall probability between $30 \%$ from the age of 65 years and 50\% from the age of 85 years. Falls are the leading cause of injury-related hospitalizations in old age and are associated with a variety of risk factors: slow reaction time, poor balance and weak muscle strength [1-3]. For targeted fall prevention to be successful, it is first necessary to identify people at risk, to introduce standardized and reliable assessment measures and, then, to put appro- 
priate interventions in place. Various fall risk screening tools have been developed and tested with moderate accuracy. These assessments often rely on self-reports (i.e. questionnaires), have low precision (i.e. stopwatch time), are expensive (i.e. require specialized equipment) or provide a one-time snapshot under ideal circumstances [4]. Recent advances in sensor technologies hold promise for task-specific assessments with greater accuracy that can be performed regularly by older people at home or while undertaking daily activities [5].

The sit-to-stand test with five repetitions [five-timessit-to-stand (5STS) test] is a functional test that is commonly used in clinical settings to assess fall risk $[6,7]$. The person is asked to stand up and sit down from a chair five times as quickly as possible with their arms folded. Previous studies have shown that performance on the 5STS is associated with reduced lower-extremity muscle strength $[8,9]$, impaired balance [9-11], reduced reaction time and psychological factors such as pain and vitality [9]. Studies have shown that the 5STS performance is slower in frailer populations and people with balance disorders [12]. Performance is usually measured in seconds, as the total stopwatch-measured 5STS time from the initial seated position to the final seated position after completing five stands. A 5STS time longer than $12-15 \mathrm{~s}$ has been associated with a higher risk of falling $[6,13]$, but there is no general agreement on the threshold that should be used to identify fallers.

The use of sensor technologies can provide a more detailed analysis of the movements during the 5STS which might help to improve the identification of fallers. Recent research has used inertial sensors, especially wearable accelerometers and gyroscopes, to quantify sit-to-stand performance in the laboratory [14-16]. The new generation of inertial and optical sensors (e.g. Microsoft Kinect) is small, portable and inexpensive and can easily be used also outside of the laboratory. Previously, the Kinect has demonstrated good accuracy compared to a gold standard Vicon motion analysis system for the 5STS test [17].

As part of this study, we examined the feasibility of the Microsoft Kinect, a low-cost, portable and marker-free computer vision sensor in a directed routine 5STS test ('Kinect-based 5STS'). We hypothesized that a detailed analysis of the participants' movements during the Kinect-based 5STS discriminates well between fallers and nonfallers and will show stronger associations with established clinical fall risk measures when compared to the standard stopwatch-measured 5STS time. Secondly, we investigated whether the Kinect-based 5STS can be used for supervised and unsupervised in-home assessments.

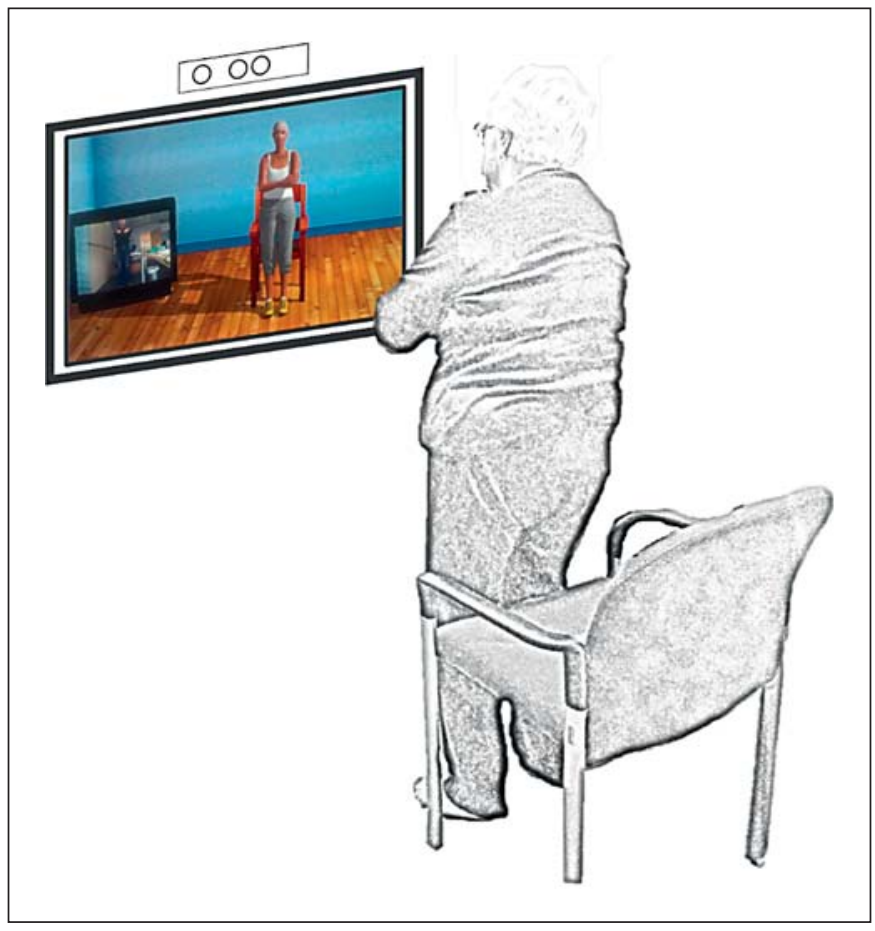

Fig. 1. Illustration of the Kinect-based 5STS.

\section{Methods}

\section{Participants}

A total of 94 community-dwelling older adults living in retirement villages in Sydney, N.S.W., Australia, participated in this study. The sample was drawn from two randomized controlled trials; 41 people were control group participants in the SureStep interactive step training trial (Australian New Zealand Clinical Trial ID: ACTRN12613000671763) and 53 were control or intervention group participants in the iStoppFalls trial (ACTRN12614000096651) [18]. The in-home assessments were conducted with the iStoppFalls intervention participants as part of the larger iStoppFalls trial [18]. The inclusion criteria were: living in the community, aged 65 years or older and being ambulant with or without the use of a walking aid. The exclusion criteria were: being medically unstable, suffering from major cognitive impairment (Mini-Cog <3), neurodegenerative disease or color blindness. Written informed consent was obtained from all participants prior to data collection. The study was approved by the University of New South Wales Human Studies Ethics Committee.

\section{Kinect-Based 5STS}

The Kinect-based 5STS relies on motion capture technology to assess performance on the 5STS. When conducting the test, participants saw themselves represented as an avatar in a virtual test environment on a television screen, and their movements were synchronously reflected by the avatar movements (fig. 1). The system consists of a personal computer, the Microsoft Kinect sensor for Windows and the assessment software. The software program 
Fig. 2. Detailed analysis of the 5STS phases (head tracking point) recorded with the Microsoft Kinect.

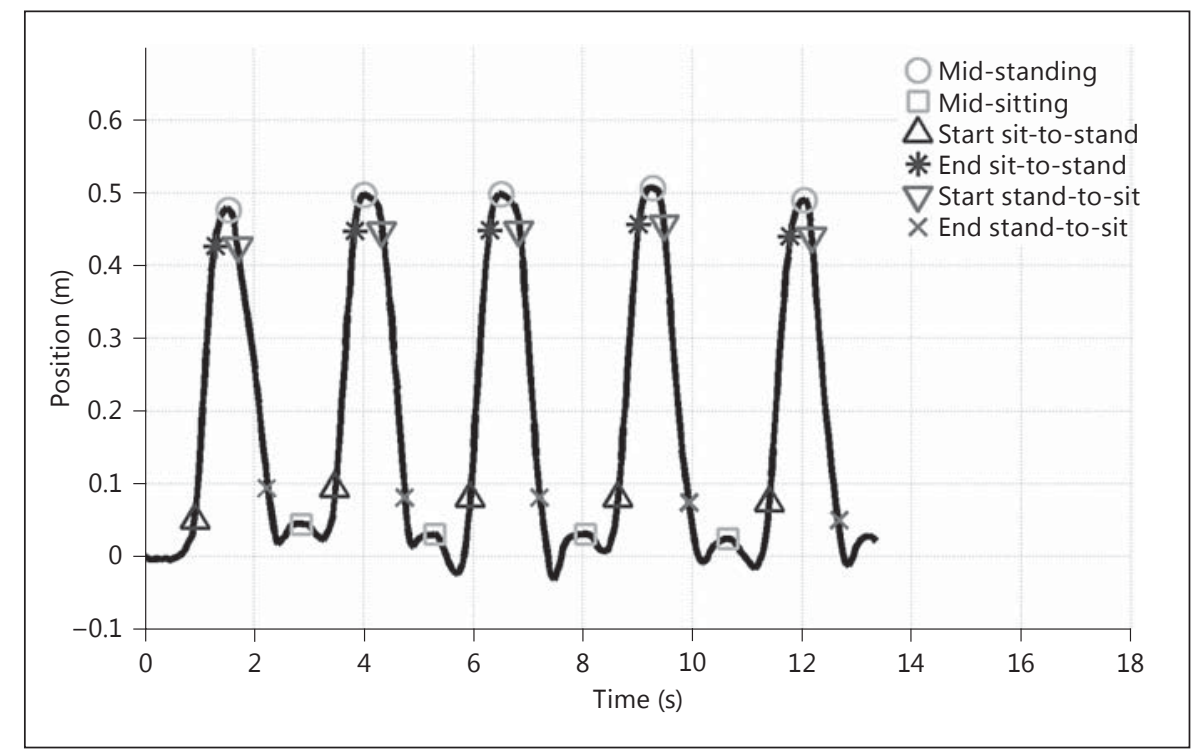

supports the test procedure by providing visual test instructions and a test start countdown. Furthermore, it displays the avatar in the virtual test environment during the test and stores the Kinect data to the personal computer.

\section{Laboratory Assessment}

Participants were asked to stand up and sit down from a standard-height chair $(45 \mathrm{~cm})$ five times as quickly as possible with their arms folded across their chest. Movements during the 5STS were recorded with the Microsoft Kinect sensor; the total stopwatch-measured 5STS time was documented for comparison. The assessments were video recorded with two standard video cameras (i.e. front and side view) to assist with the interpretation of any unusual patterns in the data during the data analysis process. The Physiological Profile Assessment (PPA) was used to estimate the overall fall risk based on tests which assess sensorimotor abilities: balance (sway when standing on a medium-density foam with eyes open), lower-extremity muscle strength (knee extension), hand reaction time, contrast sensitivity (Melbourne Edge Test) and peripheral sensation (proprioception) [19]. A medical history was recorded during a face-to-face interview, including medications, the presence of medical conditions and number of self-reported falls in the past 12 months. A fall was defined as 'an unexpected event in which the person comes to rest on the ground, floor, or lower level' [20].

\section{In-Home Assessment}

The Kinect-based 5STS was conducted in the homes of a subsample of 20 participants ( 8 fallers, 12 nonfallers) by a trained researcher (i.e. supervised assessment). The time between baseline (laboratory) testing and the in-home supervised assessment was on average $40( \pm 20)$ days. Participants were asked to perform the 5STS unsupervised within the first 30 days after installing the system. For this study, the correlations between (1) the laboratory and supervised in-home assessment and (2) the laboratory and first unsupervised in-home assessment were analyzed.

\section{Data Acquisition and Analysis}

The Microsoft Kinect is a marker-free computer vision sensor that can measure three-dimensional motion of a person. The Kinect sensor was placed in front of the television screen at a height of $80 \mathrm{~cm}$ and a distance of $200 \mathrm{~cm}$ from the participant. Skeleton data of anatomical landmarks in world coordinates were recorded using the Kinect Software Development Kit for Windows with a frequency of $30 \mathrm{~Hz}$ and a resolution of $640 \times 480$ pixels.

Microsoft Kinect data from the vertical displacement of the head movement were used to quantify sit-to-stand performance (fig. 2). The offset of the data was corrected by using the coordinates of the head tracking point during the start of the test as the initial position. The global start and end point of the 5STS was detected as the first vertical increase above the empirically found threshold of $5 \mathrm{~cm}$ and the last decrease below $5 \mathrm{~cm}$, respectively. A sit-to-stand cycle was split up into four phases: sitting, sit-to-stand transition, standing and stand-to-sit transition phase. A similar threshold-based method to that described by Doheny et al. [21] for accelerometer signals was used to detect the phases of the linear displacement data of the 5STS.

Our algorithm used three steps to automatically quantify 5STS performance (fig. 2):

(1) Identification of the mid-standing and mid-sitting points: the signal (head position) was low-pass filtered using a 4th-order Butterworth filter with a cutoff frequency of $2 \mathrm{~Hz}$. Mid-standing and mid-sitting positions were defined by the peak and trough, respectively.

(2) Identification of the start and end points of sitting and standing phases: the standing phase started when the signal amplitude first crossed a threshold $5 \mathrm{~cm}$ below the mid-standing point and ended with the second crossing. The sitting phase was similarly defined by a head position within $5 \mathrm{~cm}$ of the mid-sitting point.

(3) Feature extraction: a set of timing- and speed-related measurements was derived from the sensor signal. Total time of the Kinect-based 5STS, mean duration of the sitting and standing phases and the mean vertical velocity of the sit-to-stand and stand-to-sit transitions were calculated. 
Table 1. Characteristics of fallers and nonfallers

\begin{tabular}{lccc}
\hline Parameters & $\begin{array}{l}\text { Fallers } \\
(\mathrm{n}=29)\end{array}$ & $\begin{array}{l}\text { Nonfallers } \\
(\mathrm{n}=65)\end{array}$ & $\mathrm{p}$ value \\
\hline Age, years & $80.6 \pm 6.7$ & $79.3 \pm 6.3$ & 0.369 \\
Height, cm & $159.2 \pm 8.1$ & $163.1 \pm 9.1$ & 0.055 \\
Weight, kg & $67.2 \pm 10.8$ & $71.6 \pm 13.7$ & 0.135 \\
Body mass index & $26.5 \pm 3.2$ & $26.9 \pm 4.6$ & 0.680 \\
\hline
\end{tabular}

\section{Statistical Analysis}

Analysis of covariance (ANCOVA) was used to evaluate differences between the faller and nonfaller groups adjusted for participants' height. Cohen's d values were calculated to obtain measures of effect size. Pearson's correlation coefficients were calculated to quantify the association between the supervised laboratory, supervised and unsupervised in-home assessments and between the 5STS and clinical fall risk measures. Correlation results were categorized as weak $(0.1-0.3)$, moderate $(0.4-0.6)$ and strong $(0.7-$ $0.9)$ after the schema from Dancey and Reidy [22]. The paired t test was used to test for significant differences between the laboratory and in-home assessments. $p$ values $<0.05$ were considered to be statistically significant. Signal processing and statistical data analysis were performed in MATLAB 8.2 (R2013b).

\section{Results}

The participants $(\mathrm{n}=94)$ were $79.7 \pm 6.4$ years old; 66 (70\%) women participated in this study. Twenty-nine (31\%) people were classified as fallers with one or more falls in the past 12 months. There were no significant differences in age, weight and body mass index between the fallers and nonfallers. The differences in height were close to significance (table 1).

\section{Laboratory Assessment}

Fallers were significantly slower than nonfallers on Kinect-based measures and the total stopwatch-measured 5STS time (table 2). The mean velocity of the sit-to-stand transitions was the best discriminator between the fallers and nonfallers with an effect (d) of 0.67. This effect size was comparable to the one of the PPA $(\mathrm{d}=0.65)$, a multicomponent clinical fall risk assessment.

The correlations between the Kinect-based measures and the strength (i.e. knee extension), balance (i.e. sway when standing on a medium-density foam with eyes open) and reaction time (i.e. hand reaction time) components of the PPA were weak to moderate (table 3 ). The mean velocity of the sit-to-stand transitions was the only measure that was significantly correlated to all three PPA measures, with a stronger correlation to the knee extension strength compared to the other performance indicators of the 5STS.

\section{In-Home Assessment}

The supervised in-home assessment (i.e. Kinect-based 5STS) was conducted by 20 participants. One person was not able to perform the 5STS with arms folded and one recording had to be excluded because of technical issues. On average, the 18 participants completed the test in $11.01 \pm 2.79 \mathrm{~s}$ in their homes compared with $13.42 \pm$ $5.30 \mathrm{~s}$ in the laboratory $(\mathrm{p}=0.008)$. The correlations between the Kinect-based 5STS assessments in the laboratory and the supervised in-home assessments for the total time $(\mathrm{r}=0.832, \mathrm{p}<0.001)$, sitting time $(\mathrm{r}=0.824$, $\mathrm{p}<0.001)$, standing time $(\mathrm{r}=0.758, \mathrm{p}<0.001)$, sit-tostand velocity $(\mathrm{r}=0.704, \mathrm{p}=0.001)$ and stand-to-sit velocity $(r=0.777, p<0.001)$ were strong. Figure 3 illustrates the comparison of the mean sit-to-stand velocity for the two assessments.

Thirteen participants ( 6 fallers, 7 nonfallers) performed the unsupervised 5STS on their own. Three participants had to be excluded from further data analysis because of data recording issues. Two participants performed four instead of five repetitions and, therefore, only their mean sit-to-stand velocity measurements were included in the analysis. Participants completed the 5STS in $11.82 \pm 2.04 \mathrm{~s}$ in the laboratory and in $9.70 \pm 1.39 \mathrm{~s}$ independently in their homes $(\mathrm{p}<0.001)$. The correlations between the laboratory and the unsupervised in-home assessments for the total time $(\mathrm{r}=0.922, \mathrm{p}=0.001)$, sitting time $(\mathrm{r}=0.845, \mathrm{p}=0.008)$, standing time $(\mathrm{r}=0.775, \mathrm{p}=$ $0.024)$, sit-to-stand velocity $(\mathrm{r}=0.931, \mathrm{p}=0.002)$ and stand-to-sit velocity $(\mathrm{r}=0.820, \mathrm{p}=0.013)$ were strong. Figure 3 shows this relationship for the mean sit-to-stand velocity. Participants reported no falls or other adverse events in relation to undertaking the Kinect-based 5STS test.

\section{Discussion}

In this study, the feasibility of the Kinect-based 5STS for fall risk assessment in community-living older people was examined. To our knowledge, this is the first study which has used the Kinect sensor to assess 5STS performance in the laboratory and home setting in older people.

The present findings showed that an automated and more detailed analysis of the 5STS is feasible. Our algorithm was able to correctly identify each phase and to 

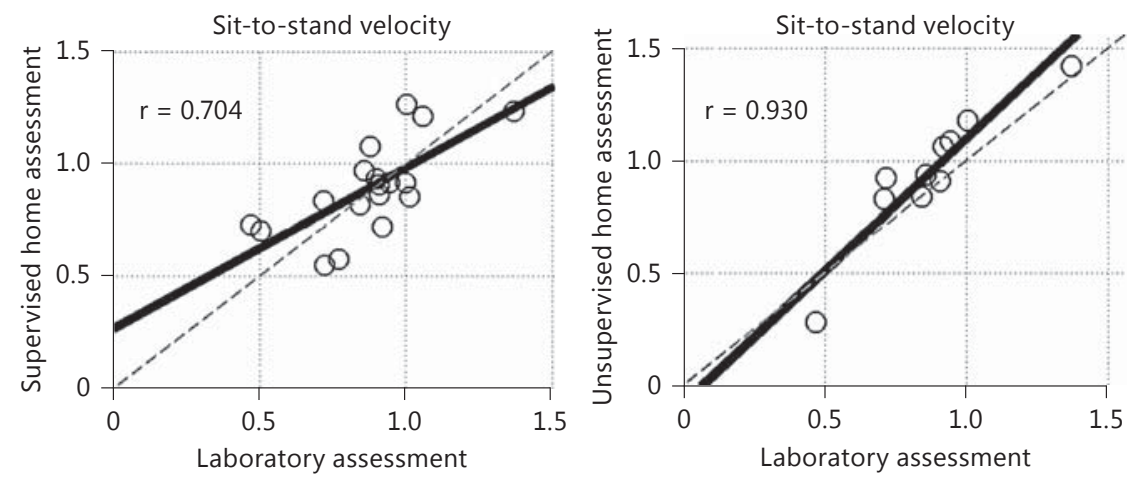

Fig. 3. Correlations between the laboratory and supervised, and laboratory and unsupervised home assessments for the mean sit-to-stand velocity.

Table 2. Test scores for the clinical and Kinect-based 5STS assessments for the fallers and nonfallers

\begin{tabular}{lcccc}
\hline Measurement & Fallers $(\mathrm{n}=29)$ & Nonfallers $(\mathrm{n}=65)$ & $\mathrm{p}$ value & $|\mathrm{d}|$ \\
\hline Clinical fall risk measures & & & & \\
$\quad$ Stopwatch-measured 5STS time, s & $16.80 \pm 5.68$ & $14.33 \pm 4.53$ & $0.028^{*}$ & 0.50 \\
$\quad$ PPA score & $1.73 \pm 0.87$ & $1.18 \pm 0.83$ & $0.014^{*}$ & 0.65 \\
Kinect-based 5STS measures & & & \\
$\quad$ Total time, s & $15.33 \pm 5.45$ & $13.12 \pm 4.06$ & $0.034^{*}$ & 0.49 \\
Mean sitting time, s & $1.75 \pm 0.88$ & $1.46 \pm 0.59$ & 0.071 & 0.42 \\
Mean standing time, s & $1.02 \pm 0.38$ & $0.85 \pm 0.31$ & 0.063 & 0.51 \\
Mean sit-to-stand velocity, m/s & $0.78 \pm 0.20$ & $0.94 \pm 0.24$ & $0.019^{*}$ & 0.67 \\
Mean stand-to-sit velocity, m/s & $0.65 \pm 0.20$ & $0.76 \pm 0.22$ & 0.151 & 0.48 \\
\hline$* \mathrm{p}<0.05$. & & & & \\
\hline
\end{tabular}

Table 3. Correlations between the Kinect-based 5STS measures and the strength, balance and reaction time components of the PPA

\begin{tabular}{llccc}
\hline Measurement & Stopwatch 5STS & Strength & Balance & Reaction time \\
\hline Clinical fall risk measures & & & & \\
$\quad$ Stopwatch-measured 5STS time (s) & - & $-0.317^{* *}$ & $0.255^{*}$ & 0.188 \\
Kinect-based 5STS measures & & & & \\
$\quad$ Total time (s) & $0.994^{* *}$ & $-0.316^{* *}$ & $0.234^{*}$ & 0.167 \\
$\quad$ Mean sitting time (s) & $0.904^{* *}$ & $-0.304^{* *}$ & $0.252^{*}$ & 0.055 \\
$\quad$ Mean standing time (s) & $0.874^{* *}$ & $-0.326^{* *}$ & 0.161 & $0.285^{* *}$ \\
$\quad$ Mean sit-to-stand velocity (m/s) & $-0.586^{* *}$ & $0.533^{* *}$ & $-0.246^{*}$ & $-0.321^{* *}$ \\
$\quad$ Mean stand-to-sit velocity (m/s) & $-0.517^{* *}$ & $0.432^{* *}$ & -0.078 & $-0.274^{* *}$ \\
\hline$* \mathrm{p}<0.05 ; * * \mathrm{p}<0.01$. & & & & \\
\hline
\end{tabular}


extract time- and speed-related measures from the sensor signals of all successfully completed tests. The mean sit-to-stand velocity classified fallers and nonfallers well based on 12-month retrospective fall data. Furthermore, it had stronger associations with clinical tests for balance, strength and reaction time than the stopwatch-measured 5STS time. Noteworthy, the correlation between the sitto-stand velocity and the stopwatch-measured 5STS time itself was only moderate, which supports the hypothesis that the sit-to-stand velocity, as a performance indicator for muscle power, is more than just an alternate measurement of total STS time. Our findings are in accordance with the study by Doheny et al. [21] who used accelerometers and concluded that a more detailed analysis of the 5STS phases provides an improved discrimination of fallers from nonfallers compared to the stopwatch-measured 5STS time. In addition, our findings demonstrate good criterion validity indicated by good correlations between the in-home and laboratory Kinect-based 5STS measures.

We acknowledge certain study limitations, most of which relate to the pioneering use of a new technology. First, while a history of falls has been shown to be a good predictor of future falls, the retrospective recording of falls may have underestimated the true prevalence of fall events. Further studies are warranted to assess the predictive power of the Kinect-based 5STS for future falls. Second, the assessments were not standardized for time of day or attire. Third, the avatar-based approach might have influenced participants' timing of the 5STS. In the supervised settings, participants were instructed to focus on the task rather than on the movements of the avatar. While it was feasible to administer the Kinect-based 5STS in a supervised home setting, only $56 \%$ of the participants performed the Kinect-based 5STS unsupervised within 30 days. Participants reported technical issues with the system, especially at the beginning of the study, which might have prevented them from turning the Kinectbased 5STS on. The Microsoft Kinect is also sensitive to different light conditions and sometimes fails to locate and isolate the participant if objects (e.g. a chair) are in close proximity. Further studies are warranted to assess the feasibility of the unsupervised Kinect-based 5STS. Based on informal user feedback, it appears that unsupervised assessments would be improved by (1) less complex system designs, (2) clearer test instructions, (3) more reliable equipment and (4) more engaging test assessments (e.g. gamification). Lastly, we acknowledge that an unsupervised Kinect-based 5STS might not be feasible in people with higher levels of frailty.
In future, the Kinect-based 5STS could be used in clinical settings as an objective, inexpensive and quick test to identify older people at increased risk of falls. With further development, in-home assessments could be conducted by the following means: (1) test administered on regular home visits by trained personnel or (2) test performed independently and unsupervised as a self-assessment. Both in-home methods would provide measurements of fall risk over time and could be used to provide feedback regarding physical benefits (e.g. of a fall prevention exercise program). This feedback could be in written or visual form and accessible through the television for older people. Further, the Kinect-based 5STS system could be connected to the internet. This would enable clinicians to access the results remotely and the system could automatically generate alerts regarding significant improvements or declines.

In summary, our findings indicate that the Kinectbased 5STS discriminated well between fallers and nonfallers and was feasible to administer in clinical and supervised in-home settings. This study represents an important step towards the development of home-based fall risk assessments. With further research, especially under unsupervised conditions, the assessments may prove useful as a fall risk screen and home-based assessment measure of functional mobility for monitoring changes over time as well as the effects of fall prevention interventions.

\section{Acknowledgment}

The Kinect-based 5STS was developed as part of the iStoppFalls project, an EU-funded (grant agreement 287361) initiative for fall prevention and prediction (iStoppFalls: An EU Fall Prevention Project; http://www.istoppfalls.eu). Stephen R. Lord is an NHMRC Senior Principal Research Fellow, Kim Delbaere is an NHMRC Career Development Fellow and Matthew Brodie is an NHMRC Early Career Development Fellow. Yves J. Gschwind was funded by the Margarethe and Walter Lichtenstein Foundation, Switzerland. We would like to thank our research assistants and participants.

$\begin{array}{ll}\text { References } \quad 1 \text { Ejupi A, Brodie M, Gschwind YJ, Schoene D, } \\ \\ \text { Lord SR, Delbaere K: Choice Stepping Reac- } \\ \text { tion Time test using Exergame technology for } \\ & \text { fall risk assessment in older people. Conf Proc } \\ & \text { IEEE Eng Med Biol Soc 2014;2014:6957- } \\ & 6960 . \\ 2 & \text { Tinetti ME, Kumar C: The patient who falls: } \\ & \text { 'It's always a trade-off. JAMA 2010;303:258- } \\ & 266 .\end{array}$


3 Delbaere K, Jacqueline CT, Heim J, Perminder SS, Brodaty H: A multifactorial approach to understanding fall risk in older people. J Am Geriatr Soc 2010;58:1679-1685.

4 Howcroft J, Kofman J, Lemaire ED: Review of fall risk assessment in geriatric populations using inertial sensors. J Neuroeng Rehabil 2013;10:91.

5 Ejupi A, Lord SR, Delbaere K: New methods for fall risk prediction. Curr Opin Clin Nutr Metab Care 2014;17:407-411.

6 Tiedemann A, Shimada H, Sherrington C, Murray S, Lord SR: The comparative ability of eight functional mobility tests for predicting falls in community-dwelling older people. Age Ageing 2008;37:430-435.

7 Macrae PG, Lacourse M, Moldavon R: Physical performance measures that predict faller status in community-dwelling older adults. J Orthop Sports Phys Ther 1992;16:123-128.

8 Csuka M, McCarty DJ: Simple method for measurement of lower extremity muscle strength. Am J Med 1985;78:77-81.

9 Lord SR, Murray SM, Chapman K, Munro B, Tiedemann A: Sit-to-stand performance depends on sensation, speed, balance, and psychological status in addition to strength in older people. J Gerontol A Biol Sci Med Sci 2002;57:539-543.

10 Whitney SL, Wrisley DM, Marchetti GF, Gee MA, Redfern MS, Furman JM: Clinical measurement of sit-to-stand performance in people with balance disorders: validity of data for the Five-Times-Sit-to-Stand Test. Phys Ther 2005;85:1034-1045.
11 Sherrington C, Lord SR, Close JCT, Barraclough E, Taylor M, O'Rourke S, Kurrle S, Tiedemann A, Cumming RG, Herbert RD: Development of a tool for prediction of falls in rehabilitation settings (Predict_FIRST): a prospective cohort study. J Rehabil Med 2010; 42:482-488.

12 Whitney SL, Wrisley DM, Marchetti GF, Gee MA, Redfern MS, Furman JM: Clinical measurement of sit-to-stand performance in people with balance disorders: validity of data for the Five-Times-Sit-to-Stand Test. Phys Ther 2005;85:1034-1045.

13 Buatois S, Miljkovic D, Manckoundia P, Gueguen R, Miget P, Vançon G, Perrin P, Benetos A: Five times sit to stand test is a predictor of recurrent falls in healthy community-living subjects aged 65 and older. J Am Geriatr Soc 2008;56:1575-1577.

14 Van Lummel RC, Ainsworth E, Lindemann U, Zijlstra W, Chiari L, Van Campen P, Hausdorff JM: Automated approach for quantifying the repeated sit-to-stand using one body fixed sensor in young and older adults. Gait Posture 2013;38:153-156.

15 Doheny EP, Walsh C, Foran T, Greene BR, Wei C, Cunningham C, Anne R: Falls classification using tri-axial accelerometers during the five-times-sit-to-stand test. Gait Posture 2013;38:1021-1025.
16 Zijlstra W, Bisseling RW, Schlumbohm S, Baldus $\mathrm{H}$ : A body-fixed-sensor-based analysis of power during sit-to-stand movements. Gait Posture 2010;31:272-278.

17 Galna B, Barry G, Jackson D, Mhiripiri D, Olivier P, Rochester L: Accuracy of the Microsoft Kinect sensor for measuring movement in people with Parkinson's disease. Gait Posture 2014;39:1062-1068.

18 Gschwind YJ, Eichberg S, Marston HR, Ejupi A, De Rosario H, Kroll M, Drobics M, Annegarn J, Wieching R, Lord SR, Aal K, Delbaere K: ICT-based system to predict and prevent falls (iStoppFalls): study protocol for an international multicenter randomized controlled trial. BMC Geriatr 2014;14:91.

19 Lord SR, Menz HB, Tiedemann A: A physiological profile approach to falls risk assessment and prevention. Phys Ther 2003;83: 237-252.

20 Lamb SE, Jørstad-Stein EC, Hauer K, Becker C: Development of a common outcome data set for fall injury prevention trials: the Prevention of Falls Network Europe consensus. J Am Geriatr Soc 2005; $53: 1618-1622$

21 Doheny EP, Fan CW, Foran T, Greene BR, Cunningham C, Kenny RA: An instrumented sit-to-stand test used to examine differences between older fallers and non-fallers. Conf Proc IEEE Eng Med Biol Soc 2011;2011:30633066.

22 Dancey C, Reidy J: Statistics without Maths for Psychology: Using SPSS for Windows. London, Prentice Hall, 2004. 